\title{
Efficiency of spin-to-orbit conversion in crystals subjected to torsion stresses
}

\author{
Vasylkiv Yu., Skab I. and Vlokh R. \\ Institute of Physical Optics, 23 Dragomanov St., 79005 Lviv, Ukraine, \\ vlokh@ifo.lviv.ua
}

Received: 21.12 .2012

\begin{abstract}
We introduce a figure of merit describing the efficiency of SAM-to-OAM conversion in single crystals subjected to mechanical torsion. It is shown that the figure of merit is defined by the effective piezooptic coefficient of optical material and its refractive index. We derive the effective piezoptic coefficients for the crystals of different symmetry groups and show that, among the well-studied crystalline materials, the highest figure of merit is peculiar for the SAM-to-OAM conversion occurring in $\mathrm{LiNbO}_{3}, \beta-\mathrm{BaB}_{2} \mathrm{O}_{4}$ and $\alpha-\mathrm{BaB}_{2} \mathrm{O}_{4}$ crystals.
\end{abstract}

Keywords: orbital angular momentum, optical vortices, crystals, torsion stresses

PACS: $78.20 . \mathrm{H}-$, 42.50.Tx

UDC: 535.55

\section{Introduction}

Recently we have shown that the optical vortices with unit topological charges can be generated using torsion of solid crystals, when a crystalline sample is placed between crossed circular polarisers [1]. A torsion moment applied to the crystals containing three-fold symmetry axes [2] creates a peculiar distribution of optical indicatrix around the torque axis, which finally leads to formation of a helical mode in the emergent light beam. Axial distribution of the optical indicatrix in crystals subjected to the torsion stresses is quite similar to distribution of director orientation in so-called $q$-plates [3] typically used for a spin-to-orbit angular momentum (SAM-to-OAM) conversion.

In single solid crystals, such a distribution of the eigenvectors of optical impermeability tensor appears due to a piezooptic effect, which is induced by the shear stress components $\sigma_{13}=\sigma_{5}$ and $\sigma_{23}=\sigma_{4}$ occurring under torsion around a $Z$ axis. Then the efficiency of the SAMto-OAM conversion should depend on the piezooptic parameters of crystals. Let us notice that the efficiency of the SAM-to-OAM conversion represents a very important parameter, especially for the crystalline materials which can have separate areas of application, when compared with the $q$-plates based on liquid crystals. For example, solid crystalline materials possess wider ranges of optical transparency, higher optical damage thresholds and wider ranges of temperature stability, when compared with the liquid crystals. The present work is aimed at deriving a figure of merit describing the SAM-to-OAM conversion and selecting the most efficient crystals for generation of doughnut modes.

\section{Results and discussion}

Let us analyse the appearance of the OAM in the outgoing beam propagating through a system consisting of a right-handed circular polariser, a sample subjected to a torque moment, and a lefthanded circular analyser. The phase front of the incident beam with the radius $\rho=R$ is taken to be 
approximately plane, while the SAM of the incident photons is equal to $S^{i n c}=-\hbar$. The component of the electric field of the incident beam may be presented as

$$
E^{\text {out }}(\rho, \varphi)=E_{a} \cos \frac{\Delta \Gamma(\rho)}{2}\left[\begin{array}{c}
1 \\
\pm i
\end{array}\right]+i E_{a} \sin \frac{\Delta \Gamma(\rho)}{2} e^{ \pm i 2 q \varphi \pm i 2 \alpha_{0}}\left[\begin{array}{c}
1 \\
\mp i
\end{array}\right],
$$

where $2 q=m=1$ is the number of helicoidal lobes, $\Delta \Gamma$ the phase difference, $E_{a}$ the incident planewave amplitude, $\alpha_{0}$ the angle of optical indicatrix orientation at $\varphi=0, E^{\text {out }}(\rho, \varphi)$ the outgoing wave amplitude, and $\rho$ and $\varphi$ imply the polar coordinates. The first term in r. h. s. of Eq. (1) describes the outgoing wave with the plane front and the same SAM value as for the incident wave (i.e., $-\hbar$ ), while the second term describes a helical mode bearing the OAM (see [3]). Basing on the requirement of conservation of the total angular momentum, one can write out the relation for the angular momentum transformation:

$$
J^{\text {inc }}=J^{\text {out }}+M,
$$

where $J^{i n c}=S^{i n c}=-\hbar$ is the total angular momentum for the incident photon, $J^{\text {out }}=L^{\text {out }}+S^{\text {out }}=-2 q \hbar+\hbar=0$ the total angular momentum for the outgoing photon ( $S^{\text {out }}=+\hbar, L^{\text {out }}=-2 q \hbar$ ), and $L^{\text {out }}$ the OAM for the outgoing photon. Thus, the mechanical angular momentum transferred to the medium due to the Beth effect [4] is equal to $M=-\hbar$.

However, Eq. (2) based on the requirement of angular momentum conservation is written under the condition of $\Delta \Gamma=\pi$, though the phase difference depends on the module $\rho$ in our case. It is therefore necessary to account for the fact that the dislocation-free wave described by the first term in the r. h. s. of Eq. (1) with the SAM equal to $-\hbar$ also exists in the light beam emerging a sample. Then the efficiency of the SAM-to-OAM conversion should be written as

$$
\eta=I_{l}^{\text {out }} / I_{r}^{\text {inc }},
$$

where $I_{r}^{\text {inc }}$ is the intensity of the right-handed incident wave and $I_{l}^{\text {out }}$ the intensity of the lefthanded outgoing wave. While calculating the intensity of the outgoing wave, let us use the Jones matrix approach. The cross section of a sample is divided into $3600(60 \times 60)$ elementary, optically uniform cells. The resulting Jones matrix for each elementary beam $(k, l=1 \ldots 60)$ acquires the form

$$
T^{k l}=\left|\begin{array}{cc}
e^{i \Delta \Gamma^{k l} / 2} \cos ^{2} \zeta_{Z^{\prime}}^{k l}+e^{\left.-i \Delta \Lambda^{[l / 2} \sin ^{2} \zeta_{Z^{\prime}}^{k l}\right)} & i \sin \left(\Delta \Gamma^{k l} / 2\right) \sin 2 \zeta_{Z^{\prime}}^{k l} \\
i \sin \left(\Delta \Gamma^{k l} / 2\right) \sin 2 \zeta_{Z^{\prime}}^{k l} & \left(e^{i \Delta \Gamma^{k l} / 2} \sin ^{2} \zeta_{Z^{\prime}}^{k l}+e^{-i \Delta \Gamma^{k l} / 2} \cos ^{2} \zeta_{Z^{\prime}}^{k l}\right)
\end{array}\right|,
$$

where $\quad \sigma_{4}^{k l}=\frac{2 M_{Z^{\prime}}}{\pi R^{4}}(k-30) \times 10^{-4}, \quad \sigma_{5}^{k l}=\frac{2 M_{Z^{\prime}}}{\pi R^{4}}(l-30) \times 10^{-4}, \quad \Delta \Gamma^{k l}=2 \pi d_{Z^{\prime}} \Delta n_{X^{\prime} Y^{\prime}} / \lambda, \quad$ and $\zeta_{Z^{\prime}}^{k l}=\frac{1}{2} \arctan \frac{\sigma_{4}^{k l}}{\sigma_{5}^{k l}}$ (with $\Delta n_{X^{\prime} Y^{\prime}}$ and $\zeta_{Z^{\prime}}^{k l}$ being respectively the induced birefringence and the optical indicatrix rotation - see [2]). Here the $X^{\prime} Y^{\prime} Z^{\prime}$ coordinate system coincides with the principal axes of the Fresnel ellipsoid in the case of optically uniaxial crystals, and it is rotated with respect to the crystallographic system of the cubic crystals in such a way that the $Z^{\prime}$ axis is parallel to the [111] direction. The components of the Jones vectors of the outgoing and incoming waves are $E_{1}, E_{2}$ and $E_{1}^{k l}, E_{2}^{k l}$, respectively. 
The Jones matrices of the orthogonally rotated quarter-wave plates are respectively $T^{Q W P-}$ and $T^{Q W P+}$, whereas the Jones matrix of the analyser is $T^{4}$. Thus, one can write

$$
\left|\begin{array}{c}
E_{1}^{k l} \\
E_{2}^{k l}
\end{array}\right|=T^{A} T^{Q W P-} T^{k l} T^{Q W P+}\left|\begin{array}{c}
E_{1} \\
E_{2}
\end{array}\right|,
$$

where

$$
\begin{aligned}
& E_{1}=1, \quad E_{2}=0, \quad T^{A}=\left(\begin{array}{ll}
0 & 0 \\
0 & 1
\end{array}\right), \\
& T^{Q W P-}=\left(\begin{array}{l}
\frac{1}{\sqrt{2}} e^{i \frac{\pi}{4}} \frac{1}{\sqrt{2}} e^{-i \frac{\pi}{4}} \\
\frac{1}{\sqrt{2}} e^{-i \frac{\pi}{4}} \frac{1}{\sqrt{2}} e^{i \frac{\pi}{4}}
\end{array}\right), \quad T^{Q W P+}=\left(\begin{array}{l}
\frac{1}{\sqrt{2}} e^{-i \frac{\pi}{4}} \frac{1}{\sqrt{2}} e^{i \frac{\pi}{4}} \\
\frac{1}{\sqrt{2}} e^{i \frac{\pi}{4}} \frac{1}{\sqrt{2}} e^{-i \frac{\pi}{4}}
\end{array}\right) .
\end{aligned}
$$

Finally, the phase difference for each of the elementary beams is given by

$$
\Phi^{k l}=\arctan \left(\frac{\operatorname{Im} E_{1}^{k l}}{\operatorname{Re} E_{1}^{k l}}\right)-\arctan \left(\frac{\operatorname{Im} E_{2}^{k l}}{\operatorname{Re} E_{2}^{k l}}\right) .
$$

Notice that here the phase difference means a difference of phases of linearly polarised waves with the polarization vector directions parallel to the directions of light wave oscillations in the linear polarisers (i.e., the intensity of the wave with the polarization parallel to the direction of oscillation in the first polariser in the outgoing beam is equal to zero). Using Eq. (5), one can write out the relation for the intensity $\left(I_{l}^{\text {out }}\right)^{k l}$ of the elementary beam propagating through the optical system at the point with the coordinates $k$ and $l$ in the $X Y$ plane:

$$
\left(I_{l}^{\text {out }}\right)^{k l}=\left|E_{1}^{k l}\right|^{2}+\left|E_{2}^{k l}\right|^{2}
$$

where $E_{1}^{k l}$ and $E_{2}^{k l}$ are the components of the Jones vector of the outgoing elementary beam with the coordinates $k$ and $l$.

For numerical calculation of the SAM-to-OAM conversion efficiency, we have divided the beam into the same $k \times l(k, l=1 \ldots \mathrm{N})$ elementary beams, while the intensity of the incoming wave has been chosen to be equal to $I_{r}^{i n c}=1$. Hence, Eq. (3) may be rewritten as

$$
\eta=\frac{\sum_{k=1}^{N} \sum_{l=1}^{N}\left(I_{l}^{\text {out }}\right)^{k l}}{N^{2}} .
$$

It follows from the symmetry conditions [2] that the OAM can be generated under torsion of crystals that have three-fold axes, while the torque-moment axis and the wave vector of the incident wave should be parallel to these axes. Only in this case a canonical vortex can be generated, with the appearance of purely screw dislocation in the wave front. Such crystals should belong to the point groups of symmetry $\mathrm{m} 3 \mathrm{~m}, \mathrm{~m} 3,23,432, \overline{4} 3 \mathrm{~m}, 3 \mathrm{~m}, \overline{3} \mathrm{~m}, 32,3$, and $\overline{3}$. Following from this condition, we have studied the crystals belonging to the above point symmetry groups, for which the necessary piezooptic parameters are known form the literature. For our best knowledge, $\mathrm{LiNbO}_{3}$ [5], $\alpha-\mathrm{BaB}_{2} \mathrm{O}_{4}$ [6] and $\beta-\mathrm{BaB}_{2} \mathrm{O}_{4}$ [7], quartz [8], 
hexamethylenetetramine [9], $\mathrm{NaCl}, \mathrm{KAl}\left(\mathrm{SO}_{4}\right)_{2} \times 12 \mathrm{H}_{2} \mathrm{O}$ [9] and lead germanate [10] belong to this group of crystals.

The group could be divided into four piezooptic subgroups: (1) m3m, 432 and $\overline{4} 3 \mathrm{~m}$; (2) 23 and $\mathrm{m} 3$; (3) $3 \mathrm{~m}, \overline{3} \mathrm{~m}$ and 32 ; and (4) 3 and $\overline{3}$. These subgroups differ by their piezooptic tensors. In case when the torsion moment is applied around the optic axis in optically uniaxial crystals or around the [111] direction in the cubic crystals and the optical beam propagates along the same directions, the induced birefringence is determined by the formulae [2] $\Delta n_{X^{\prime} Y^{\prime}}=-\frac{2 \sqrt{2} n_{0}^{3}\left(\pi_{11}-\pi_{44}-\pi_{12}\right) M_{Z^{\prime}}}{3 \pi R^{4}} \sqrt{X^{\prime 2}+Y^{\prime 2}}, \quad \Delta n_{X^{\prime} Y^{\prime}}=-\frac{2 n_{0}^{3} M_{Z^{\prime}} \sqrt{\pi_{14}^{\prime 2}+\pi_{15}^{\prime 2}}}{\pi R^{4}} \sqrt{X^{\prime 2}+Y^{\prime 2}}$, with $\pi_{14}^{\prime}=\frac{1}{3}\left(\pi_{11}-\pi_{44}\right)-\frac{1+\sqrt{3}}{6} \pi_{12}-\frac{1-\sqrt{3}}{6} \pi_{21} \quad$ and $\quad \pi_{15}^{\prime}=-\frac{1}{3}\left(\pi_{11}-\pi_{44}\right)+\frac{1-\sqrt{3}}{6} \pi_{12}+\frac{1+\sqrt{3}}{6} \pi_{21}$, $\Delta n_{X Y}=2 n_{o}^{3} \frac{M_{Z}}{\pi R^{4}} \pi_{14} \sqrt{X^{2}+Y^{2}}$ and $\Delta n_{X Y}=2 n_{o}^{3} \frac{M_{Z}}{\pi R^{4}} \sqrt{\left(\pi_{14}^{2}+\pi_{25}^{2}\right)\left(X^{2}+Y^{2}\right)}$ for the first, second, third and fourth subgroups, respectively. All of these formulae are very similar and differ only by the value of the effective piezooptic coefficients. The relations for the latter coefficients are presented in Table 1 for all of the piezooptic subgroups listed above.

We have also obtained dependences of the efficiency of the SAM-to-OAM conversion on the torque moment. This has been done for cylindrical crystalline samples with the cylinder radius equal to $R=2.5 \mathrm{~mm}$ and its length $d=15 \mathrm{~mm}$. The other parameters in need, such as the ordinary refractive index $\left(n_{o}\right)$ and the optical wavelength $(\lambda)$ are presented in Table 1. For the trigonal crystals, the axis of the torsion moment and the wave vector are parallel to the optic axis, while in the cubic crystals to the direction [111]. The torsion moment $M_{Z}$ has been changed in the region of $0-0.2 \mathrm{~N} \times \mathrm{m}$. As a result, we have obtained the dependences for all of the above crystals (see Fig. 1). As seen from Fig. 1, the efficiency reaches the values as high as $\eta \sim 80 \%$ under the torsion moment $\sim 0.06 \mathrm{~N} \times \mathrm{m}$.

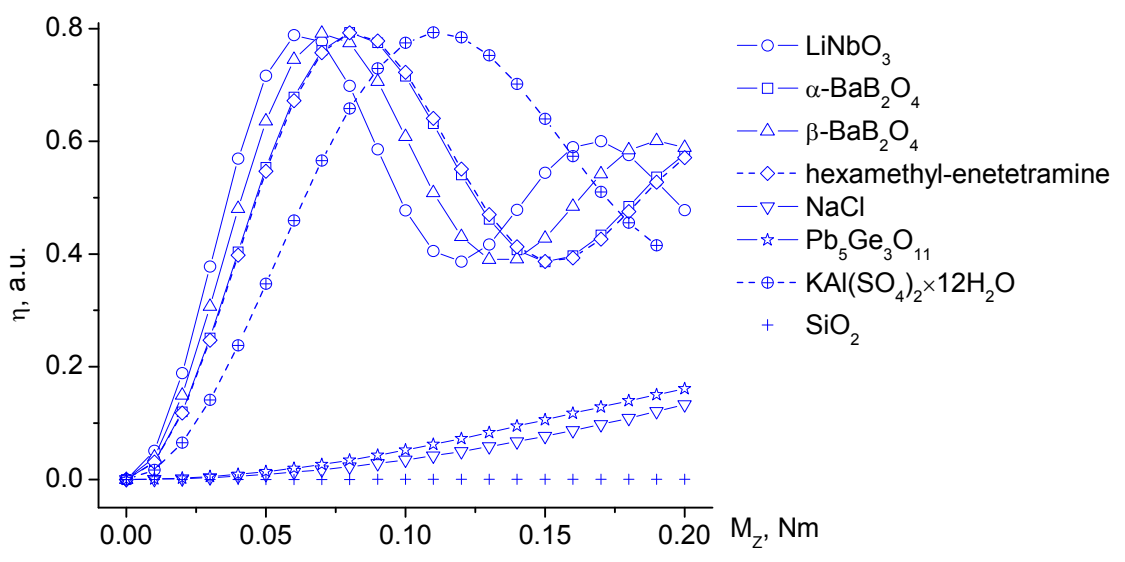

Fig. 1. Dependences of efficiency of the SAM-to-OAM conversion upon the torsion moment for different crystals.

The maximum $\eta$ values that can be reached in the crystals under consideration are collected in Table 1. As shown in the wok [11], the presence of natural optical activity leads to decreasing efficiency of the SAM-to-OAM conversion. It is indeed seen from Table 1 and Fig. 1 that the optically active crystals such as $\mathrm{SiO}_{2}$ and $\mathrm{Pb}_{5} \mathrm{Ge}_{3} \mathrm{O}_{11}$ reveal comparatively low $\eta$ parameters. 
Table 1. Parameters defining the efficiency of SAM-to-OAM conversion under crystal torsion.

\begin{tabular}{|c|c|c|c|c|c|c|c|}
\hline $\begin{array}{l}\text { Crystal } \\
\text { (point } \\
\text { symmetry } \\
\text { group) }\end{array}$ & $\begin{array}{c}\text { Piezooptic } \\
\text { coefficient } \pi_{i \mu} \\
\left(10^{-12} \mathrm{~m}^{2} / \mathrm{N}\right) \text { and } \\
\text { gyration tensor } \\
\text { component } \\
\end{array}$ & Relation for $\pi_{i \mu}^{e f f}$ & $\begin{array}{l}\text { Wave- } \\
\text { length } \\
\lambda, \mathrm{nm}\end{array}$ & $\begin{array}{l}\text { Refrac- } \\
\text { tive } \\
\text { index } n_{o}\end{array}$ & $\begin{array}{l}\eta, \\
\text { a. u. }\end{array}$ & $\begin{array}{c}M_{Z}, \\
\mathrm{~N} \times \mathrm{m}\end{array}$ & $\begin{array}{c}W \\
10^{-12} \\
\mathrm{~m}^{2} / \mathrm{N}\end{array}$ \\
\hline $\begin{array}{l}\mathrm{LiNbO}_{3} \\
(3 m)\end{array}$ & $\begin{array}{c}\pi_{i \mu}^{e f f}=\pi_{14}=0.887 \\
{[5]}\end{array}$ & $\pi_{14}$ & 632.8 & 2.28647 & 0.8 & 0.06 & 10.6 \\
\hline $\begin{array}{c}\beta-\mathrm{BaB}_{2} \mathrm{O}_{4} \\
(3 m)\end{array}$ & $\begin{array}{c}\pi_{i \mu}^{e f f}=\pi_{14}=-2.0 \\
{[7]}\end{array}$ & $\pi_{14}$ & 632.8 & 1.6712 & 0.8 & 0.07 & 9.3 \\
\hline $\begin{array}{c}\alpha-\mathrm{BaB}_{2} \mathrm{O}_{4} \\
(\overline{3} m)\end{array}$ & $\begin{array}{c}\pi_{i \mu}^{e f f}=\pi_{14}=1.77 \\
{[6]}\end{array}$ & $\pi_{14}$ & 632.8 & 1.6738 & 0.8 & 0.08 & 8.3 \\
\hline $\begin{array}{l}\mathrm{SiO}_{2} \\
(32)\end{array}$ & $\begin{array}{l}\pi_{i \mu}^{\text {eff }}=\pi_{14}=-0.11 \\
g_{3}=12.9 \times 10^{-5}\end{array}$ & $\pi_{14}$ & 589 & 1.5442 & 0.0003 & 0.2 & 0.4 \\
\hline $\begin{array}{c}\mathrm{Pb}_{5} \mathrm{Ge}_{3} \mathrm{O}_{11} \\
\text { (3) }\end{array}$ & $\begin{array}{l}\pi_{i \mu}^{e f f}=\sqrt{\pi_{14}^{2}+\pi_{25}^{2}} \\
\quad=0.35[10], \\
g_{3}=4.02 \times 10^{-5}[8]\end{array}$ & $\sqrt{\pi_{14}^{2}+\pi_{25}^{2}}$ & 632.8 & 2.115 & 0.16 & 0.2 & 3.3 \\
\hline $\begin{array}{l}\mathrm{NaCl} \\
(m 3 m)\end{array}$ & $\begin{array}{c}\pi_{11}=1.27 \\
\pi_{12}=2.58 \\
\pi_{44}=-0.84[9] \\
\pi_{i \mu}^{e f f}=-0.22\end{array}$ & $\begin{array}{l}\frac{\sqrt{2}}{3} \times \\
\left(\pi_{11}-\pi_{44}-\pi_{12}\right)\end{array}$ & 589 & 1.5443 & 0.1 & 0.2 & 0.8 \\
\hline $\begin{array}{c}\mathrm{KAl}\left(\mathrm{SO}_{4}\right)_{2} \times \\
12 \mathrm{H}_{2} \mathrm{O} \\
(m 3)\end{array}$ & $\begin{array}{c}\pi_{11}=2.43, \\
\pi_{12}=6.66 \\
\pi_{21}=6.99 \\
\pi_{44}=-0.55[9], \\
\pi_{i \mu}^{\text {eff }}=1.82\end{array}$ & $\sqrt{\pi_{14}^{\prime 2}+\pi_{15}^{\prime 2}} *$ & 589 & 1.4564 & 0.8 & 0.11 & 5.6 \\
\hline $\begin{array}{l}\text { hexame- } \\
\text { thylene- } \\
\text { tetramine } \\
(\overline{4} 3 \mathrm{~m})\end{array}$ & $\begin{array}{c}\pi_{11}=11.95 \\
\pi_{12}=8.42 \\
\pi_{44}=7.56[9] \\
\pi_{i \mu}^{\text {eff }}=-1.9\end{array}$ & $\begin{array}{l}\frac{\sqrt{2}}{3} \times \\
\left(\pi_{11}-\pi_{44}-\pi_{12}\right)\end{array}$ & 589 & 1.591 & 0.8 & 0.08 & 7.7 \\
\hline
\end{tabular}

* $\pi_{14}^{\prime}=\frac{1}{3}\left(\pi_{11}-\pi_{44}\right)-\frac{1+\sqrt{3}}{6} \pi_{12}-\frac{1-\sqrt{3}}{6} \pi_{21}$ and $\pi_{15}^{\prime}=-\frac{1}{3}\left(\pi_{11}-\pi_{44}\right)+\frac{1-\sqrt{3}}{6} \pi_{12}+\frac{1+\sqrt{3}}{6} \pi_{21}$

For a particular case when the beam propagates through a system of orthogonal circular polarisers and a crystalline sample subjected to a torque moment is placed in between, Eq. (8) may be written as

$$
\left(I_{l}^{\text {out }}\right)^{k l}=\left(I_{r}^{i n c}\right)^{k l} \sin ^{2}(\Delta \Gamma / 2)
$$

under the condition that the induced phase difference for each beam is constant. Then the efficiency of the SAM-to-OAM conversion given by Eq. (9), with accounting for the relations for the effective piezooptic coefficient (see Table 1), is determined as follows:

$$
\eta=\left.\frac{\sum_{X} \sum_{Y} \sin ^{2}\left(W \frac{d_{Z}}{\lambda} \frac{M_{Z}}{R^{4}} \sqrt{X^{2}+Y^{2}}\right)}{N^{2}}\right|_{X^{2}+Y^{2} \leq R^{2}},
$$

where $W=n_{0}^{3} \pi_{i \mu}^{e f f}$ is the parameter that defines the efficiency of the SAM-to-OAM conversion 
(i.e., the figure of merit of the SAM-to-OAM conversion) and $\pi_{i \mu}^{e f f}$ represents the effective piezooptic coefficient. As a consequence, searching for efficient materials for the SAM-to-OAM conversion occurring under crystal torsion is reduced to searching for crystalline materials with the trigonal or cubic symmetries, which reveal high figures of merit $W$. We have found that the highest figure of merit for the crystals under consideration is typical for the lithium niobate (see Table 1). A comparable $W$ coefficient is peculiar of the borate crystals (e.g., $\beta-\mathrm{BaB}_{2} \mathrm{O}_{4}$ and $\alpha-\mathrm{BaB}_{2} \mathrm{O}_{4}$ ), as well as hexamethylenetetramine and $\mathrm{KAl}\left(\mathrm{SO}_{4}\right)_{2} \times 12 \mathrm{H}_{2} \mathrm{O}$ crystals. Notice also that the borate crystals are resistant to high-power optical radiation and. moreover, they are transparent in the deep ultraviolet spectral range.

\section{Conclusions}

In the present work we have introduced a parameter that determines the efficiency of SAM-toOAM conversion associated with the torsion of single crystals. This figure of merit is given by the formula $W=n_{0}^{3} \pi_{i \mu}^{e f f}$ and is given by the effective piezooptic coefficient of optical material and its refractive index. We have derived the effective piezoptic coefficients for the crystals of different symmetry groups. We have also shown that the highest figures of merit for the SAM-to-OAM conversion are typical of the $\mathrm{LiNbO}_{3}, \beta-\mathrm{BaB}_{2} \mathrm{O}_{4}$ and $\alpha-\mathrm{BaB}_{2} \mathrm{O}_{4}$, hexamethylenetetramine and $\mathrm{KAl}\left(\mathrm{SO}_{4}\right)_{2} \times 12 \mathrm{H}_{2} \mathrm{O}$ crystals. Nonetheless, from the technological point of view the best materials for such optical applications are $\mathrm{LiNbO}_{3}, \beta-\mathrm{BaB}_{2} \mathrm{O}_{4}$ and $\alpha-\mathrm{BaB}_{2} \mathrm{O}_{4}$ crystals.

\section{Acknowledgement}

The work was supported through the grant GP/F44/002 from State Fundamental Research Fund of Ukraine.

\section{References}

1. Skab I, Vasylkiv Yu, Savaryn V and Vlokh R. 2011. Optical anisotropy induced by torsion stresses in $\mathrm{LiNbO}_{3}$ crystals: appearance of an optical vortex. J. Opt. Soc. Amer. A. 28: 633640.

2. Skab I, Vasylkiv Yu, Zapeka B, Savaryn V and Vlokh R, 2011. Appearance of singularities of optical fields under torsion of crystals containing threefold symmetry axes. J. Opt. Soc. Amer. A. 28: $1331-1340$.

3. Marrucci L, 2008. Generation of helical modes of light by spin-to-orbital angular momentum conversion in inhomogeneous liquid crystals. Mol. Cryst. Liq. Cryst. 488: 148-162.

4. Beth R A, 1936. Mechanical detection and measurement of the angular momentum of light. Phys. Rev. 50: 115-125.

5. Skab I, Smaga I, Savaryn V, Vasylkiv Yu and Vlokh R, 2011. Torsion method for measuring piezooptic coefficients. Cryst. Res. Techn. 46: 23-36.

6. Vasylkiv Yu, Savaryn V, Smaga I, Skab I and Vlokh R, 2011.On determination of sign of the piezo-optic coefficients using torsion method. Appl. Opt. 50: 2512-2518.

7. Andrushchak A S, Bobitski Ya V, Kaidan M V, Mytsyk B G, Kityk A V and Schranz W, 2005. Two-fold interferometric measurements of piezo-optic constants: application to $\beta$ - $\mathrm{BaB}_{2} \mathrm{O}_{4}$ crystals. Opt. Laser Technol. 37: 319-328.

8. Shaskolskaya M P, Acoustic crystals, Moscow: Nauka (1982).

9. Haussühl S, Physical properties of crystals: an introduction. Weinheim: Wiley-VCH Verlag $\mathrm{GmbH}$ (2008). 
10. Skab I, Vasylkiv Yu, Smaga I, Savaryn V and Vlokh R, 2011. On the method for measuring piezooptic coefficients $\pi_{25}$ and $\pi_{14}$ in the crystals belonging to point symmetry groups 3 and $\overline{3}$. Ukr. J. Phys. Opt. 12: 28-35.

11. Vasylkiv Yu, Krupych O, Skab I and Vlokh R, 2011. On the spin-to-orbit momentum conversion operated by electric field in optically active $\mathrm{Bi}_{12} \mathrm{GeO}_{20}$ crystals. Ukr. J. Phys. Opt. 12: $171-179$.

Vasylkiv Yu., Skab I. and Vlokh R., 2013. Efficiency of spin-to-orbit conversion in crystals subjected to torsion stresses. Ukr.J.Phys.Opt. 14: 50 - 56.

Анотація. В роботі отримано коефіцієнт якості, який описує ефективність спінорбітального перетворення в монокристалах під дією механічного кручення. Показано, шзо даний коефіцієнт якості визначається ефективним n'єзооптичним коефіцієнтом матеріалу та його показником заломлення. Отримані ефективні п'єзооптичні коефіцієнти для кристалів, які належать до різних груп симетрії $і$ показано, щьо серед вивчених кристалічних матеріалів найвищим значенням коефіцієнта якості володіють кристали $\mathrm{LiNbO}_{3}, \mathrm{\beta}-\mathrm{BaB}_{2} \mathrm{O}_{4} \mathrm{ma} \alpha-\mathrm{BaB}_{2} \mathrm{O}_{4}$. 